\title{
„Das Fremde“ besser verstehen
}

\author{
Kurt W. Schmidt
}

Online publiziert: 1. Juli 2015

(C) Springer-Verlag Berlin Heidelberg 2015

Im Jahr 2000 berichtet die pakistanische Ärztin und Medizinethikerin Farhat Moazam im Hastings Center Report von ihren Erfahrungen mit der Arzt-Patient-Beziehung in unterschiedlichen Ländern [7]. Ausgebildet in Pakistan und dann viele Jahre in den USA tätig, übernahm Frau Dr. Moazam eine Stelle an einer Universitätsklinik in Pakistan. Zu einer ihrer ersten ärztlichen Aufgaben gehörte es dort, mit den beiden Söhnen eines Patienten zu sprechen. Der Patient selbst litt an einer weit fortgeschrittenen Krebserkrankung und seine Lebenserwartung wurde als sehr begrenzt eingestuft. Der Patient, der bei seinem ältesten Sohn und dessen Familie lebte, war selbst bei diesem Gespräch nicht anwesend, wohl aber neben den beiden Söhnen noch die unverheiratete Tochter, eine Schwiegertochter und ein erwachsener Enkelsohn. Nachdem die Familie den Ausführungen der Ärztin gefolgt und über den geschilderten Krankheitszustand sichtlich betroffen war, ergriff der älteste Sohn das Wort und legte der Ärztin dar: „Wir möchten nicht, dass ihm sein Krankheitszustand mitgeteilt wird. Wie lange er zu leben hat, liegt in Gottes Hand und es wäre nicht richtig, meinem Vater in seinem Zustand auch noch jegliche Hoffnung zu nehmen. "Und dann fügte der Sohn noch hinzu: „Doktor Sahib, sagen Sie uns, was wir als nächstes tun sollen. Sie sind nicht nur unsere Ärztin, Sie sind für uns wie eine Mutter!“”

Die geschulte Leserin und der geschulte Leser aus dem „westlichen Kulturkreis“ mag sofort einwenden, dass der „erste Fehler“ der Ärztin gewesen ist, dieses Aufklärungsgespräch nicht mit dem Patienten selbst, sondern mit den Familienangehörigen geführt zu haben. Und Farhat Moazam führt in ihrem Bericht auch zugleich an, dass ihr das Selbstbestimmungsrecht des Patienten als fundamentalem Prinzip der westlichen Medizinethik mehr als bewusst gewesen ist. Sie gibt jedoch zu bedenken, dass in Pakistan, wie in vielen anderen „,non-Western cultures“, die Entscheidung über das gesundheitliche Wohl des Patienten

Dr. K. W. Schmidt $(\bowtie)$

Zentrum für Ethik in der Medizin, Agaplesion Markus Krankenhaus,

Wilhelm-Epstein-Str. 4,

60431 Frankfurt/M, Deutschland

E-Mail: zemmarkus@aol.com 
häufig von der Familie oder dem Arzt getroffen wird. Zudem verweist die Anrede Sahib, den arabischen Wurzeln nach ein Ehrentitel wie „Lord“, darauf, dass dem Arzt die unbestrittene Autorität zukommt, wichtige Entscheidungen in Gesundheits- und Krankheitsfragen zu treffen. Dabei wird in letzter Konsequenz von allen die Überzeugung geteilt, dass Leben und Tod letztlich nicht in der Hand der Menschen liegt, auch nicht der des Arztes, sondern bei Gott allein.

Auf den ersten Blick erscheint es als Kluft zwischen zwei Kulturen: hier die Patientenautonomie als unverrückbarer medizinethischer Eckstein säkularer westlicher Gesellschaften, in der der Patient als allein kompetenter Entscheidungsträger der Hüter und Verwalter seines eigenen Schicksals ist, dort die Eingebundenheit des Einzelnen in einen familiären Kontext und die anerkannte Delegation von Entscheidungen an einen Arzt als selbstverständlichem Mitglied der Patient-Familie-Arzt Triade unter Einbeziehung religiöser Schicksalsvorstellungen. Bei näherer Betrachtung sind die zuletzt genannten holzschnittartig beschriebenen Elemente aber auch im Westen nicht unbekannt: Familienmitglieder wollen ihre erkrankten Angehörigen vor belastenden Diagnosen schützen, erbitten vom Arzt wegweisende Entscheidungen und reagieren auf Erfahrungen der Ohnmacht gegenüber schicksalhaft erlebter Krankheitsverläufe höchst unterschiedlich und keineswegs immer areligiös. Auch haben sich verschiedene Studien und Autoren gegen den als übermächtig erlebten Einfluss (USamerikanischer) bioethischer Prinzipien im Sinne eines „modernen Imperialismus“ ausgesprochen, gerade wenn sie traditionelle Werte wie Familie durch eine als gefühl- und beziehungslos verstandene Konzentration auf die Autonomie bedroht bzw. zerstört sehen. Die besondere Bedeutung der Familie für gesundheits- und krankheitsrelevante Behandlungsentscheidungen etwa in Asien ist wiederholt beschrieben worden [8], verknüpft mit der Forderung, dass die Behandler grundsätzlich nicht unreflektiert den wörtlichen Forderungen des Patienten nachkommen sollen, sondern dessen wahren und authentischen Bedürfnissen [5]. Doch wie können diese erkannt werden?

Welche ethischen Herausforderungen in der Begegnung mit „dem Fremden“ im Gesundheitswesen bestehen, hat H. Tristram Engelhardt, Jr. in seinem fundamentalen Werk The Foundations of Bioethics im Jahr 1986 dargelegt [3]. Wohl kaum ein anderes Buch hat Ende der 1980er Jahre derart irritiert, da es den Leser in seiner konsequenten, geradezu schonungslosen Analyse mit den eigenen Werthaltungen konfrontiert und inhaltlich weit mehr als eine Auseinandersetzung mit medizinethischen Fragen darstellt, als vielmehr eine „Biopolitik“ entwirft, die auf säkular philosophischer Basis Antwort zu geben versucht auf die Frage, wie ein friedfertiges Zusammenleben von Menschen mit unterschiedlichen Wertvorstellungen in Hinblick auf Gesundheits- und Krankheitserfahrungen und Behandlungsmöglichkeiten gelingen kann. Der Fremde, dem wir in Engelhardts Buch begegnen, ist kein geringerer als „,der Nächste“, der sich im Kern als „,moral stranger“ erweist. Und einige zentrale medizinethische Fragen, etwa nach den Grenzen und Möglichkeiten kommunikativer Verständigung, dem Personsein und der personalen Identität, werden sogar an einer Stelle am Beispiel der amerikanischen TV-Serie Raumschiff Enterprise entfaltet, dessen gesamter Plot darin besteht, dass die Besatzung des Raumschiffs jahrelang im Universum unterwegs ist, ,um fremde Welten zu erkunden, die nie ein Mensch zuvor gesehen hat."Wie sich nun die Besatzung des Raumschiffs (und nicht zu vergessen der Arzt (!) Dr. McCoy als einer der zentralen Figuren), den fremden Wesen und unbekannten Erscheinungen nähert, was sie dabei als „Person“ mit Rechten und Pflichten anerkennen (und aufgrund welcher Kriterien), all dies behandelt in seiner abstrakten Zuspitzung auf das fremde Gegenüber zentrale bioethische Fragen und legt den Grundstein für eine Erfahrung, die sich in der Kunst und 
der Wissenschaft konsequent durchhält: Dem „Fremden“ zu begegnen heißt letztlich, eine Selbsterfahrung machen.

Diese - mitunter unangenehme - Erfahrung wird im klinischen Alltag häufig verdeckt. Für Unzufriedenheit und Belastung sorgen beim Zusammentreffen von Menschen aus unterschiedlichen Ländern vor allem sprachliche Probleme. Zwar bestehen diese auch in vielen anderen Situationen des Alltags, doch im Falle von Krankheit haben die unterschiedlich Betroffenen es hier mit existentiellen Nöten und Krisen zu tun, häufig verbunden mit tiefen Ängsten, die die interkulturellen und interreligiösen Problematiken schärfer hervortreten lassen. Waren diese Konflikte früher noch dadurch gekennzeichnet, dass sich eine als mehr oder weniger ,homogen“ erlebte Gruppe des medizinisch-pflegerischen Personals „fremden“ Patienten mit anderen Wertvorstellungen gegenübersah, ist dies schon lange der Situation gewichen, dass sich sprachliche, kulturelle und religiöse Verschiedenheit auch innerhalb des Behandlungsteams wiederfinden. Dabei tauchtein der wissenschaftlichen Reflexion bereits vor Jahren die Frage auf, inwiefern die Konzentration auf „die Kultur“ überhaupt angemessen ist und weiterhilft. Denn was ist überhaupt „die Kultur“? Verkürzend lässt sich sagen, dass sich hier die Theoriediskussion in den letzten 40 Jahren von der Konzentration auf einen dominanten Einfluss „,der Kultur“, die als objektiv zu bestimmende Größe verstanden wurde, verabschiedet hat, und in den Sozialwissenshaften ein sogenannter „,cultural turn“ stattgefunden hat. So bemüht man sich heute vielmehr, etwa in den Gesundheitswissenschaften, situative Ereignisse zu ,entschlüsseln“, ob in der ArztPatienten-Beziehung, in Pflegesituationen, im Erleben von Machtverhältnissen in Gesundheitsinstitutionen. Einer allgemeingültigen, objektiven Wahrnehmung von „Kultur“ wird damit eine generelle Absage erteilt; „Kultur“ wird nunmehr verstanden als ein vom Betrachter abhängiger Terminus und vor den Folgen einer unbedachten Etikettierung wird gewarnt. Wer von „Kultur“ spricht, sollte das Wort letztlich sorgsam einsetzen und wie bei einem Medikament vor der Verwendung nach Indikation, Risiken und Nebenwirkungen fragen [6]. So ist in der Pflegewissenschaft das Konzept der „Transkulturellen Kompetenz“ entstanden, um zu einer kultursensiblen Pflege zu befähigen [1].

Dabei darf nicht vergessen werden, dass das Thema interkulturelle Kommunikation vor allem in den letzten 20 Jahren in Deutschland stark rezipiert wurde [2]. Interessant - gerade auch für unser Eingangsbeispiel - sind die berühmten Arbeiten des Niederländers Geert Hofstede, der zu Beginn der 1980er Jahre IBM-Mitarbeiter in 53 verschiedenen Ländern befragt und das Konzept der vier kulturellen Dimensionen entwickelt hatte [4]. Bei aller heutigen Kritik soll zumindest referiert werden, dass in einer Kategorie die Neigung zum Individualismus der Neigung zum Kollektivismus gegenübergestellt wurde. Die USA landeten damals auf Platz 1 (= stärkste Neigung zum Individualismus) und den letzten Platz belegten Indonesien und Pakistan (= geringste Neigung zum Individualismus bzw. stärkste Neigung zum Kollektivismus). Doch wie bereits angesprochen ist die starke Konzentration auf „Kultur“ in späteren Konzepten und Modellen zurückgetreten. So betont der Medizinethnologe Michael Knipper, dass es doch vor allem um die Aufmerksamkeit und das Interesse für die Sichtweisen und Erfahrungen der Patienten geht, um ihre Bedürfnisse im Hier und Jetzt, was letztlich wichtiger ist als abstrakte Aussagen zur „Kultur“.

Vor dem gesamten Hintergrund von Migration, Flüchtlingsbewegungen und Globalisierung entsteht für die Medizinethik - und vor allem für die klinische Ethikberatung - nun die interessante Frage, ob die klassischen Modelle der Ethikberatung sensibel genug sind, um die vielfältigen situativen Besonderheiten und Herausforderungen des Klinikalltags ange- 
messen zu erfassen. So „konkurrieren“ schon seit langem im angloamerikanischen Kontext Methoden der prinzipienbasierten klinischen Ethikberatung neben bioethischer Mediation und Formen der conflict resolution. Ob sich für bestimmte Themenkonstellationen im Rahmen der klinischen Ethikberatung die besondere Methode der „Interkulturellen Mediation“ als hilfreicher erweist, weil sie in fünf Stufen alle Parteien an das bessere Verstehen des Gegenübers heranzuführen bemüht ist, wird eine Fragen unter vielen der diesjährigen Jahrestagung der Akademie für Ethik in der Medizin sein, die vom 24.-26. September 2015 in Frankfurt/M. stattfinden wird. Welche Spannungen sich durch interreligiöse Konflikte ergeben und wie sich diese auflösen lassen, wie sich das in der Pflegewissenschaft erarbeitete Konzept der „transkulturellen Kompetenz“ unter den realen Bedingungen des praktischen Alltags umsetzen lässt, um eine kultursensible Pflege zu ermöglichen, wird ebenso diskutiert werden, wie die „kulturellen Unterschiede“ zwischen den Berufsgruppen im Gesundheitswesen. Und immer wenn es gilt, ,das Fremde“ besser zu verstehen, geht es letztlich darum, sich selbst besser zu verstehen. In diesem Sinne laden wir herzlich nach Frankfurt ein.

\section{Literatur}

1. Domenig D (Hrsg) (2007) Transkulturelle Kompetenz. 2. erw. Aufl. Huber, Bern

2. Dreißig V (2005) Interkulturelle Kommunikation im Krankenhaus. Transcript, Bielefeld

3. Engelhardt HT Jr (1986) The foundations of bioethics. Oxford University Press, New York

4. Hofstede G (1980) Culture's consequences. Sage Publications, Beverly Hills

5. Hyun I (2002) Waiver of informed consent, cultural sensitivity, and the problem of unjust families and traditions. Hastings Cent Rep 32:14-22

6. Knipper M (2015) Verstehen oder Stigmatisieren? Die Krux mit der „Kultur“ in Medizin und Public Health. Public Health Forum 23:97-99

7. Moazam F (2000) Families, patients, and physicians in medical decisionmaking: a Pakistani perspective. Hastings Cent Rep 30:28-37

8. Schmidt KW, Rothärmel S, Wolfslast G (Hrsg) (2006) Familie versus Patientenautonomie? Zur Rolle der Familie bei Behandlungsentscheidungen. Haag \& Herchen, Frankfurt a. M. 